\title{
Evaluation of a Pharmacy Supported e-Cigarette Smoking Cessation Intervention in North West England
}

\author{
Alan D. Price \\ University of Salford \\ Margaret Coffey \\ University of Salford \\ Lawrence Houston \\ University of Salford \\ Penny A. Cook ( $\sim$ p.a.cook@salford.ac.uk) \\ University of Salford
}

\section{Research Article}

Keywords: Smoking cessation, electronic cigarettes, e-cigarettes, pharmacy, mixed-methods, longitudinal, community intervention.

Posted Date: November 30th, 2021

DOI: https://doi.org/10.21203/rs.3.rs-1038808/v1

License: () (1) This work is licensed under a Creative Commons Attribution 4.0 International License. Read Full License 


\section{Abstract}

Background: Cigarette smoking cessation has been described as the world's most important public health intervention. Electronic cigarettes are a relatively new tool for assisting smoking cessation but there is a lack of data on their efficacy. This article reports on a pharmacy supported e-cigarette smoking cessation intervention undertaken in a metropolitan area in the north of England.

Methods: Longitudinal mixed-methods evaluation incorporating analysis of secondary data, interviews with service users, and interviews with service providers at 3-month and 12-month follow-up, with an additional text message survey of service users at 12-month follow-up.

Results: The four-week follow-up data suggest that for every twenty people given an e-cigarette, six quit smoking tobacco and three people cut their cigarette intake by more than five cigarettes per day. Long-term follow-up results were positive but only a small number of participants were still engaged with the study at 12 months. Service users and providers spoke positively about the combination of e-cigarettes and pharmacy support.

Conclusions: E-cigarette distribution combined with pharmacy support appears to be an agreeable and effective intervention for smoking cessation, but further data are needed on long-term quit rates and health effects.

\section{Background}

Internationally, tobacco smoking is responsible for more than eight million deaths per year. ${ }^{1}$ Around seven million of these deaths are directly due to individuals smoking, while around one million are due to second-hand smoke. ${ }^{1}$ In England, around 78,000 people die each year from smoking-related illnesses, and around 490,000 are admitted to hospital due to smoking. ${ }^{2}$ Tobacco smoking not only contributes to death and mortality, but is a considerable financial cost to smokers, leading to financial difficulties and stress. ${ }^{3}$ Smoking cessation has been described as potentially the most important public health intervention globally, if effective. ${ }^{4}$ It can offer several health and financial benefits to smokers and their families, ${ }^{3,5}$ including the reduction of second-hand smoke exposure. ${ }^{5}$ However, despite many smokers reporting a desire to stop smoking, ${ }^{6}$ successful, long-term cessation is challenging. Barriers include: stress and smoking as stress management; the social acceptability of smoking especially in low-income and marginalised communities; and a lack of effective interventions, especially those involving contact with healthcare professionals ${ }^{7}$. Due in part to such barriers, rates of quitting smoking may be as low as $3 \%$, and rates of relapse may be as high as $80 \% .^{8}$

There may be a cyclical relationship between smoking and stress, whereby higher levels of stress in smokers becomes a barrier to quitting or a cause of relapsing, and the expense of smoking cigarettes creates financial stress. ${ }^{3}$ This has been found to be particularly harmful in people from lower-income backgrounds who have less disposable income and may be at risk of financial problems caused by continued spending on cigarettes. ${ }^{9}$ In the UK, likelihood of being a smoker tends to correlate with socioeconomic status; around $9.3 \%$ of people in managerial and professional occupations were smokers in 2019 , while the proportion in intermediate occupations was $14.1 \%$ and in manual and routine occupations the figure was $23.4 \% .{ }^{10} \mathrm{Smokers}$ from disadvantaged areas of society including lower income groups could benefit from short-term, individual-level interventions. 7,11

Individual-level interventions to promote smoking cessation include brief interventions, behavioural interventions, nicotine replacement therapy (NRT), pharmaceutical therapies, and combinations of these such as a behavioural intervention combined with pharmaceutical treatment. ${ }^{4,12,13}$ Brief interventions take the form of a short conversation between a healthcare practitioner and their patient or client, often lasting only a few seconds or minutes. ${ }^{12}$ There is evidence of their effectiveness, albeit at fairly low levels, but this is seen as an efficient use of time. ${ }^{14}$ Behavioral interventions typically consist of regular support meetings with a healthcare professional, and will often be combined with another form of intervention such as NRT. They can be delivered to individuals or groups over a number of weeks and may involve some form of recognised therapy such as cognitive behavioural therapy. ${ }^{12}$ Their efficacy has been difficult to assess however, because they are typically delivered alongside other interventions. A recent systematic review found stronger evidence of efficacy for brief interventions compared to longer-term behavioural interventions. ${ }^{15}$ NRT and other pharmaceutical treatments can reduce the urge to smoke by either delivering nicotine without the use of tobacco (in the case of NRT), by delivering a psychoactive compound which blocks the brain's nicotine receptors (Varenicline), or by using an antidepressant to suppress the negative emotions associated with nicotine withdrawal (Bupropion and similar drugs). ${ }^{12}$ A review of trials showed that Varenicline performs better than placebo and better than Bupropion as an aid to smoking cessation ${ }^{16}$. These and other methods of aiding smoking cessation are generally seen as a useful range of tools from which practitioners and public health bodies can choose alongside broader strategies such as bans on advertising and increased taxes on cigarettes. ${ }^{17}$ Aside from the tools used to aid smoking cessation, the use of local pharmacies in smoking cessation efforts has been shown to increase the efficacy of programmes. ${ }^{18}$ Pharmacies represent a cost-effective and efficient system of delivering evidence-based interventions alongside practitioner advice and support. ${ }^{18}$

Recently, another tool has emerged as a potentially useful and effective option to aid smoking cessation. Electronic cigarettes (e-cigarettes) are small, handheld, battery-operated devices that resemble cigarettes. They work by heating a solution containing nicotine which is then inhaled as an aerosol into the lungs of the user (known as vaping). ${ }^{19}$ As well as representing an effective method of nicotine delivery, they may also be beneficial as an aid to smoking cessation due to their hand-to-mouth and inhalation actions, and the presence of a visible vapour released upon exhalation, resembling the experience of smoking. ${ }^{4} \mathrm{E}$ cigarette users (known as vapers) in the UK have reported enjoying the experience of using their e-cigarettes, and were able to switch easily from conventional cigarettes, especially due to their effective nicotine delivery and the action of vaping as similar to that of cigarette smoking. ${ }^{20}$ Studies into the efficacy of ecigarettes as aids to smoking cessation have tended to use observational methods rather than trials, and have tended not to find that vaping leads to longterm cessation. ${ }^{19}$ Some studies have found that, rather than being useful as an aid to cessation, vapers often switch between conventional cigarettes and ecigarettes and adopt e-cigarettes as part of their smoking routine ${ }^{20}$. However, one recent randomised trial found that e-cigarettes were more effective as an aid 
to smoking cessation than NRT, when each were provided alongside behavioural support. ${ }^{21}$ E-cigarettes are not without their risks however. As well as containing nicotine, e-cigarette vapour contains other substances that are known to be harmful upon inhalation, some of which may be carcinogenic. ${ }^{22}$ There is some debate around the safety of e-cigarettes and long-term data are still needed, ${ }^{23}$ but some studies have concluded that the risks associated with vaping are minimal when compared to the well-known health risks associated with smoking conventional cigarettes, and that e-cigarettes are preferable. ${ }^{24-26}$ The UK Royal College of Physicians has endorsed the use of e-cigarettes as an aid to smoking cessation, and Public Health England have reported that e-cigarettes are around $95 \%$ less harmful than conventional cigarettes. ${ }^{23}$ Others have been more critical however, ${ }^{27}$ and some public health bodies have been less positive. For example, the US Preventive Services Task Force has reported that there is not sufficient evidence to recommend e-cigarettes as an aid to smoking cessation. ${ }^{23}$

To summarise: E-cigarettes have been shown to be a promising intervention for smoking cessation and are generally viewed as positive in the UK. Healthcare professional-assisted interventions, especially those conducted via pharmacies, have been shown to increase intervention efficacy at a relatively low cost. Smoking cigarettes is harmful to the health and finances of smokers, their families and the people around them, particularly for lower income groups such as manual and routine workers. Smokers are often motivated to stop smoking but find it difficult and interventions can increase the chance of successful quitting

In 2017, the Greater Manchester Health and Social Care Partnership (GMHSCP) 28 published a strategy to reduce adult smoking in the region to $13 \%$, or by 115,000 smokers. As part of that plan, two local authorities in Greater Manchester introduced e-cigarette interventions delivered by pharmacies. In 2018 , the first intervention ${ }^{29}$ recruited 1,022 smokers, 383 (37\%) of whom had quit smoking tobacco after four weeks. Among those still smoking tobacco, the average number of cigarettes smoked per day had dropped from 19.1 to 8.7. People in less deprived areas and working in higher-paid jobs were more likely to have quit tobacco. The study concluded that such schemes appear to be effective, but more work was needed on targeting smokers from lower income backgrounds, and interventions needed to be followed up over a longer period. Here we report on the second intervention, which used similar methods to the first programme: a pharmacy-supported smoking cessation intervention using e-cigarettes, which targeted smokers in manual and routine occupations. This study additionally included a 12 month follow up.

\section{Methods}

\section{Intervention}

Between January and June 2019, a pharmacy-supported smoking cessation intervention (funded by the local authority) was delivered in a metropolitan borough in Greater Manchester by six pharmacies. The aim of the project was to offer smoking cessation support and free e-cigarettes to around 800 residents who were routine and manual workers, as well as social housing tenants. The aims of the intervention were to:

- Support a sample of routine and manual workers to stop smoking

- Explore the effectiveness of e-cigarettes as a smoking cessation aid, together with smoking cessation support delivered by pharmacies

- Explore challenges and facilitators to pharmacies in successfully delivering smoking cessation services

\section{Design}

The evaluation consisted of a longitudinal mixed-methods evaluation of secondary data collected by the pharmacies, with phase one data collected from zero to three months, followed by a 12-month follow-up at phase two. The evaluation was designed to:

- Determine the extent to which the intervention was successful in terms of achieving smoking abstinence and/or smoking reduction across 12 months

- Explore the impact and perceived value of the project on current smokers' behaviour

- Explore the experience of delivering the project from a pharmacy perspective, including facilitators and barriers to encouraging participants to stop smoking using an e-cigarette

- Understand the experience of engaging in the project, including facilitators and barriers to quitting smoking using an e-cigarette

- Explore the impact of delivery methods and practices (intervention fidelity) across the different pharmacies to understand any impact this may have had on quit rates

\section{Participants and procedure}

\section{Phase 1}

Figure 1 shows a flow chart of participants through both stages of data collection. Service users $(n=871)$ were provided with e-cigarettes, a charger and fluids. All equipment was provided by pharmacies, who also gave advice on smoking cessation and e-cigarette use. The intervention period was three months, and routinely collected data were taken from service users at baseline, 2 weeks and 4 weeks (endpoint). Data were collected by pharmacies on service user demographics (including age, gender, occupation and Index of Multiple Deprivation (IMD), previous use of e-cigarettes, smoking status, carbon monoxide (CO) readings (to corroborate self-reported smoking status), and data related to e-cigarette fluid provision (e.g. flavour and strengths). After three months, a sample of service users $(n=26)$ were interviewed by the research team to discuss their participation in the project, especially in terms of the approach, the impact of the intervention on their smoking behaviour, and any barriers or facilitators to quitting cigarettes. Recruitment to interviews was discontinued when thematic saturation was reached. Interviews were conducted face-to-face or by telephone. Interviews were also held with one member of staff from five out of the six service providers $(n=5)$, to discuss their experience of delivering the intervention, approaches that were taken to recruitment and delivery, the effectiveness of 
those approaches, their perceived impact on smoking behaviour, and barriers and facilitators to encouraging smokers to quit smoking using an e-cigarette. One service provider declined to take part in the interviews.

\section{Phase 2}

After 12 months, text messages were sent to all service users with a valid phone number on record and who had provided consent to be contacted. A total of 466 text messages were sent; 355 were successfully delivered and 77 service users returned surveys. As shown in figure 2 , service users were asked about their current use of cigarettes and e-cigarettes, the strength of nicotine used in e-cigarettes, and about any support they were receiving. They were also asked whether they would consent to an interview. Following this, interviews were conducted with all consenting service users ( $n=15$ ), where they were asked about their cigarette and e-cigarette use since the intervention, their experience of the intervention and the support they received from the service providers, and about any other sources of support post-intervention. Interviews were also held with service providers $(n=4)$, who were asked about their experience of the intervention, any service users who had kept in touch, perceived barriers and facilitators to quitting or reducing cigarette smoking, and their thoughts on potential future interventions. Two service providers declined to take part in the interviews.

\section{Analysis}

Quantitative data were analysed descriptively. Characteristics of quitters were compared to those who did not quit using chi square tests. Qualitative data were analysed thematically, using the methods recommended by Braun and Clarke. ${ }^{30}$

\section{Results}

\section{Phase 1 (3-month evaluation): secondary data}

As shown in figure 1, a total of 871 service users were provided with e-cigarettes and advice from one of the pharmacies involved in the intervention. Service users were invited to present back to the pharmacy after four weeks, and those who did not attend were contacted by SMS (text message). At this stage, 401 service users were unresponsive and lost to phase one follow-up. Of the remaining 470 service users, 184 ( $21.1 \%$ of those originally enrolled) were confirmed as having quit smoking tobacco via self-report along with a negative carbon monoxide reading (less than five parts per million). A further 83 ( $9.5 \%$ ) service users reported that they had stopped smoking tobacco, but either did not present for a CO test or were CO positive when tested (greater than five parts per million). A total of $203(23.3 \%)$ service users reported that they had not stopped smoking tobacco at this stage.

Table 1 shows a comparison between those who were and were not smoking tobacco at four-week follow-up. Confirmed and unconfirmed quitters are grouped together (quit), and those who were lost to follow-up were assumed to be still smoking tobacco and are grouped together with the self-reported smokers (not quit). The overall quit rate was $267(30.7 \%)$. There was no significant difference in quit rate based on age or gender. Service users who identified as white (27.8\%) were less likely to have quit than those who identified as non-white or preferred not to state their ethnicity (44.6\%). There was significant quit variation between occupational groups; those in the managerial and professional occupations were most likely to quit (40.0\%), while those who were unemployed were the least likely (20.5\%). Similarly, quit rates were the lowest in the most deprived quintile (25\%) and highest in the least deprived (40\%). However, it should be noted that, due to the targeting of the intervention in the areas of most need, the absolute numbers of quitters was higher in the most deprived quintile (73 individuals) than in the two most affluent quintiles (24 individuals). Finally, there was also a significant effect of baseline smoking category, where the lightest smokers were most likely to quit (37\%) and the heaviest smokers least likely (26\%). 
Table 1

Comparison of baseline characteristics of those who quit tobacco at four-week follow-up with

those still using tobacco (or lost to follow-up*) at four-week follow-up (phase 1)

\begin{tabular}{|c|c|c|c|c|c|c|}
\hline & Quit N (\%) & Not quit N (\%) & Total & Chi & df & $P$ \\
\hline \multicolumn{7}{|l|}{ Gender } \\
\hline Female & $140(28.6)$ & $349(71.4)$ & 489 & 0.70 & 1 & 0.402 \\
\hline Male & $116(31.3)$ & $255(68.7)$ & 371 & & & \\
\hline \multicolumn{7}{|l|}{ Age group } \\
\hline $18-24$ & $17(21.3)$ & $63(78.8)$ & 80 & 4.76 & 5 & 0.446 \\
\hline $25-34$ & $51(27.3)$ & $136(72.7)$ & 187 & & & \\
\hline $35-44$ & $47(31.5)$ & $102(68.5)$ & 149 & & & \\
\hline $45-54$ & $70(32.9)$ & $143(67.1)$ & 213 & & & \\
\hline $55-64$ & $49(30.1)$ & $114(69.9)$ & 163 & & & \\
\hline $65+$ & $22(32.4)$ & $46(67.6)$ & 68 & & & \\
\hline \multicolumn{7}{|l|}{ Ethnicity } \\
\hline White & $211(27.8)$ & $548(72.2)$ & 759 & 12.0 & 1 & 0.001 \\
\hline Non-white/prefer not to say & $45(44.6)$ & $56(55.4)$ & 101 & & & \\
\hline \multicolumn{7}{|l|}{ Occupational status } \\
\hline 1 Unemployed & $54(20.5)$ & $210(79.5)$ & 264 & 21.6 & 6 & 0.001 \\
\hline 2 Home Carer & $12(24.5)$ & $37(75.5)$ & 49 & & & \\
\hline 3 Managerial and Professional & $40(40.0)$ & $60(60.0)$ & 100 & & & \\
\hline 4 Intermediate & $34(37.4)$ & $57(62.6)$ & 91 & & & \\
\hline 5 Routine and Manual & $68(34.2)$ & $131(65.8)$ & 199 & & & \\
\hline 6 Retired & $29(33.0)$ & $59(67.0)$ & 88 & & & \\
\hline 7 Sick or Disabled & $19(27.5)$ & $50(72.5)$ & 69 & & & \\
\hline \multicolumn{7}{|l|}{ IMD Quintile } \\
\hline Most deprived & $73(24.9)$ & $220(75.1)$ & 293 & 11.3 & 4 & 0.023 \\
\hline 2 & $53(26.8)$ & $145(73.2)$ & 198 & & & \\
\hline 3 & $68(36.6)$ & $118(63.4)$ & 186 & & & \\
\hline 4 & $34(30.6)$ & $77(69.4)$ & 111 & & & \\
\hline Least deprived & $24(40.0)$ & $36(60.0)$ & 60 & & & \\
\hline \multicolumn{7}{|l|}{ Baseline category of smoking } \\
\hline Lowest (1-10) & $72(36.5)$ & $125(63.5)$ & 197 & 8.1 & 2 & 0.018 \\
\hline Medium (11-19) & $78(32.5)$ & $162(67.5)$ & 240 & & & \\
\hline Highest (20+) & $102(25.8)$ & $294(74.2)$ & 396 & & & \\
\hline Missing & 4 & 23 & 27 & & & \\
\hline Total & $256(29.8)$ & $604(70.2)$ & 860 & & & \\
\hline
\end{tabular}

Importantly, even those who reported still smoking tobacco ('non quits') recorded having halved the number of cigarettes smoked (from 19.3 to 8.7 cigarettes per day, $n=178$ ) and halved the level of CO recorded (15.4 to $8.6 \mathrm{ppm}, \mathrm{n}=104)$. Sixty-one percent of the 'non-quitters' had reduced their cigarette consumption by more than 5 cigarettes a day.

\section{Phase 1 interviews with service users}

The evaluation found six key themes in relation to service users' experiences of taking part in the e-cigarette project. Supporting quotes are shown in Table 2. 


\begin{tabular}{|c|c|}
\hline Theme & Example supporting quotes \\
\hline $\begin{array}{l}\text { Quality of } \\
\text { service } \\
\text { provided }\end{array}$ & $\begin{array}{l}\text { "Well it was excellent, they rang me when I needed to go in for a check you know and to refill the liquid, because they gave them free of } \\
\text { charge and they did a } \mathrm{CO} 2 \text { reading, to check the smoke in the blood, in the lungs and what have you, and he was very, very nice the man } \\
\text { that was there to see me." (Female; dual-use*). }\end{array}$ \\
\hline \multirow{2}{*}{$\begin{array}{l}\text { Health and } \\
\text { financial } \\
\text { benefits of e- } \\
\text { cigarette use }\end{array}$} & $\begin{array}{l}\text { "I think it's a very good idea, I really do. Yeah, let's put it like this, at the time, my chest and my breathing was really, really, really bad, } \\
\text { from smoking cigarettes, even though I didn't smoke that many, and then I started on the e-cigarette, I still haven't cut them out } 100 \%, \text { I've } \\
\text { cut them out about } 95 \% \text {, but this e-cigarette it's just like, it's saved my life if you like." (Male; dual-use). }\end{array}$ \\
\hline & $\begin{array}{l}\text { "Yeah definitely and it's not only saved me money, from not buying cigarettes, but it's saved me money from initially getting into my } \\
\text { vaping. You know because it was a free service so." (Male; dual use }{ }^{\star} \text { ). }\end{array}$ \\
\hline $\begin{array}{l}\text { Effectiveness } \\
\text { of e-cigarette }\end{array}$ & $\begin{array}{l}\text { "Well it takes the craving away definitely. I believe really, it's just because you're using your hands and your kind of imitating smoking." } \\
\text { (Male; quit). }\end{array}$ \\
\hline Mental health & $\begin{array}{l}\text { "I started to use it but then, unfortunately my mum got poorly and has passed away so, I don't think it's been the right time for me to } \\
\text { completely... I probably wouldn't be smoking, I would have carried on using it, but because of my circumstances, my mind frame wasn't } \\
\text { in it." (Female: smoker). }\end{array}$ \\
\hline $\begin{array}{l}\text { Beginning the } \\
\text { quit journey } \\
\text { using an e- } \\
\text { cigarette }\end{array}$ & $\begin{array}{l}\text { "When I first started with it, I was choking a lot, where obviously I wasn't used to it, but once you get used to it, it's basically a simple } \\
\text { object to use. But it's just trying to get used to it all." (Female, dual-use*) }\end{array}$ \\
\hline $\begin{array}{l}\text { Concerns over } \\
\text { e-cigarette use }\end{array}$ & $\begin{array}{l}\text { "It's, technically you're burning oil and inhaling the vapours off oil, so that surely, that's got to have some sort of effect on your lungs, or } \\
\text { in the long term you know?" (Male; dual-use*). }\end{array}$ \\
\hline
\end{tabular}

* dual use $=$ smoking tobacco and vaping

\section{Quality of service provided}

Service users reported a high level of satisfaction in the service they received from the providers. Many discussed receiving useful, detailed advice about the ecigarettes and how to use them. This seems to have been a key facilitator in helping to maintain the effective use of e-cigarettes. They also reported high levels of satisfaction with the quality of the devices, which were seen as superior to devices that some service users had tried in the past. Higher quality of ecigarettes was also seen as conducive to maintaining e-cigarette use and staying off tobacco. For many participants, the devices used in this study were seen as otherwise difficult to afford, so the intervention was an opportunity to step up to that higher level of quality. Participants also noted that the scheme was well advertised; they had heard about it from multiple sources including word of mouth, from a GP or nurse, an advertisement on social media or in a local newspaper, or at their local pharmacy.

\section{Health and financial benefits of e-cigarette use}

Participants described health and financial benefits of switching from tobacco to e-cigarettes. Use of the devices along with cutting down on tobacco was seen as having a noticeable improvement on health, especially in terms of respiratory problems. This was seen as leading to improvements in everyday life. In terms of the financial benefits, participants reported economic savings from buying less tobacco, and in terms of the devices being free as part of the scheme, which was gratefully accepted. A combination of health and financial benefits was seen as being a major incentive to use e-cigarettes instead of tobacco.

\section{Effectiveness of e-cigarettes}

Participants largely reported that the e-cigarettes were an effective tool for helping to reduce, and in some cases stop, tobacco use. Some noted that the handto-mouth action that resembles cigarette smoking was a useful mechanism. Criticisms were minor, but some participants noted that the resemblance was not perfect - they contrasted the 'hit' from smoking tobacco with the less noticeable effect of vaping.

\section{Mental health}

Mental health and tobacco smoking were seen as closely related. When participants were experiencing times of stress, they found it more difficult to stop smoking tobacco, even when vaping was an option. Stress or anxiety were described as triggers that led to increased tobacco smoking or decreased interest in stopping. This was sometimes seen as temporary - for example, when experiencing a bereavement, one participant described how she felt it was not the right time to stop smoking.

\section{Beginning the quit journey using an e-cigarette}

Participants described an 'adjustment phase', where some time was needed to get used to the feel of using the e-cigarettes. This was partly to do with finding a pleasing flavour and strength of liquid. There was a certain amount of trial and error involved, and it sometimes took a few different combinations until the participant were happy with the outcome. There was also an adjustment to the feel of the e-cigarette, which was different from smoking tobacco. Some participants reported side-effects such as coughing or that the sensation of inhaling vapour was unpleasant at first. However, these issues were described as temporary. Two participants also reported technical problems with their devices which rendered them unusable.

\section{Concerns over e-cigarette use}

Many participants expressed concerns over potential health impacts of vaping. The inhalation of vapour into the lungs was seen as harmful. The lack of certainty around any long-term impacts was concerning to some. Although they recognised the well-established risks of smoking tobacco, the unknown risks associated with vaping were worrying. 


\section{Phase 1 interviews with service providers}

Service providers described their experience of delivering the project; its impact on participants' smoking behaviour; programme facilitators and barriers for participants stopping smoking using the e-cigarette; and personal facilitators and barriers for quitting smoking. Supporting quotes are shown in Table 3.

\section{Pharmacists' experience of delivering the intervention}

Most of the pharmacists reported that the intervention was positive and effective. It was seen as successful in terms of helping people to stop smoking tobacco, and in some cases people who pharmacists felt would likely struggle, managed to quit. The success of the intervention was motivating for the pharmacists, who were keen for the scheme to continue. The use of an e-cigarette intervention specifically was seen as more effective than schemes using other tools, such as nicotine patches or gum. The key to this was the fact that vaping resembles tobacco smoking to a large extent.

\section{Pharmacist perceptions of the impact of the e-cigarette intervention}

Pharmacists described apparent age-related differences in the characteristics of participants. There was a feeling that younger participants were less likely to quit tobacco; even though they were using the e-cigarettes, the pharmacists reported that younger people were still smoking as well. Middle-aged and older service users were seen as more likely to quit tobacco. More broadly, pharmacists noted that the intervention was having positive effects on quitting, with even heavy and long-term smokers managing to cut down or quit. They estimated that around $50-70 \%$ of participants were managing to completely come off tobacco. Pharmacists described hearing about participants' improved health, especially related to breathing and energy levels, and about the money reportedly saved. One group of participants were apparently planning to go on holiday using the money they had saved.

Table 3

Themes and supporting quotes for phase 1 service provider interviews

\begin{tabular}{|c|c|}
\hline Theme & Example supporting quotes \\
\hline $\begin{array}{l}\text { Experience of delivering the } \\
\text { project }\end{array}$ & $\begin{array}{l}\text { "People are more excited about this one and we've seen a lot of people who've been smoking for years who've actually } \\
\text { given up, which makes us feel like we're helping" (Pharmacy 5) }\end{array}$ \\
\hline $\begin{array}{l}\text { Pharmacist perceptions of } \\
\text { the impact of the e-cigarette } \\
\text { intervention }\end{array}$ & $\begin{array}{l}\text { "...we've got quite a lot of people that have given up and they've still given up, you know even though it might be two or } \\
\text { three months later down the line... so for those people obviously a huge impact because you had people smoking you know } \\
40 \text { cigarettes a day" (Pharmacy 3). }\end{array}$ \\
\hline $\begin{array}{l}\text { Programme facilitators to } \\
\text { delivering the e-cig } \\
\text { intervention }\end{array}$ & $\begin{array}{l}\text { "There was advertising. They were advertising in the local paper, we have advertising in store and on our windows and also } \\
\text { in the local surgery...and I believe it went in the Manchester Evening News website as well" (Pharmacy 1). }\end{array}$ \\
\hline $\begin{array}{l}\text { Programme barriers to } \\
\text { delivering the intervention }\end{array}$ & $\begin{array}{l}\text { I think there's been a spectrum of impact, I think there's been quite a few people who have just got the e-cig because it's free } \\
\text { and not really you know tried it to some extent, that... weren't really invested and they've not done typically well" (Pharmacy } \\
\text { 3). }\end{array}$ \\
\hline \multirow{2}{*}{$\begin{array}{l}\text { Personal facilitators and } \\
\text { barriers for quitting smoking }\end{array}$} & “I think sometimes it's helpful if they’ve got demonstrable health problems that are linked to their smoking” (Pharmacy 3). \\
\hline & $\begin{array}{l}\text { "I think also the stress that they go through...life stress.... we do have a lot of low-income people, I think...and it boils up, you } \\
\text { know...they still want to have a cigarette when they're going through the stress, instead of having another puff on the e-cig" } \\
\text { (Pharmacist 1). }\end{array}$ \\
\hline
\end{tabular}

\section{Programme facilitators to delivering the e-cig intervention}

Pharmacists felt that the success of the scheme, in terms of reaching high numbers of people, was largely down to effective and widespread advertising. The formal scheme advertising conducted through newspapers and in pharmacies apparently continued to spread substantially by word of mouth. The fact that free e-cigarettes were being offered was popular with the public, so the nature of the scheme may have been the mechanism for continuing word-of-mouth spread. Another facilitator of success was that the intervention was conducted in a relaxed high-street setting rather than a more clinical doctors' office or hospital. The pharmacy consultations were seen as highly productive; this was seen as an opportunity to give tailored advice to service users and support and encourage them during the quitting process. For example, some service users talked about still smoking the occasional cigarette and this was seen as a failure, but pharmacists were able to re-frame this as merely a lapse or a bump along the road towards quitting. The e-cigarettes themselves were seen as helpful toward quitting, especially since they were seen as a new and advanced technology. This was especially true of the bigger Arc Slim variety, which had longer lasting batteries and adjustable strength. The use of a carbon monoxide monitor at the pharmacies was described as a useful way of motivating service users to quit. It was capable of both negative and positive reinforcement depending on the result. Finally, pharmacists reported being well-supported by the Local Authority in terms of responding to questions and replacing stock.

\section{Programme barriers to delivering the intervention}

Pharmacists highlighted a number of potential barriers to the success of the project, which were at least relevant to some participants. Barriers tended to fall into one of four categories: a lack of commitment from some service users; the short timeframe of the study; difficulties related to products and ordering; and a lack of referrals from primary healthcare providers.

Some service users seemed to lose interest after they were supplied with their equipment. Many service users were not able to be contacted after this point, with phone numbers that were out of service. This was especially common in people who were not previously known to the pharmacist. Whilst advertising of the scheme was successful at least in terms of numbers, pharmacists felt that some service users did not understand the nature of the intervention - people seemed to be aware of the e-cigarette giveaway but not the consultations and carbon monoxide testing. The four-week timeframe of the intervention was 
described as too short on two counts: one because it meant high demand by a large number or people over a short space of time, putting pressure on the staff; and two because four weeks was not seen as sufficient time for smokers to switch from tobacco to e-cigarettes. Finally, there were some difficulties with the availability and delivery of additional liquids to the pharmacies.

\section{Personal facilitators and barriers for quitting smoking}

Pharmacists were able to identify a number of personal (to the service users) facilitators and barriers to quitting tobacco. Service users were seen as more likely to cut down or quit if they used their e-cigarette regularly as a means of overcoming the urge to smoke. People who had existing health problems were seen as being more highly motivated to quit in order to improve their health. People who had other people around them who were involved in the programme and traying to quit were seen as more likely to succeed, with one pharmacist noting that some groups of men formed competitions with one another, and so had a competitive motivator to quitting. Service users with higher levels of stress in their lives were seen as less likely to quit smoking, as were those with other drug or alcohol dependence and mental health issues. Having access to cigarettes was also noted as a factor - if people were in an environment (at work or at home) with cigarettes available, they were seen as more likely to smoke.

\section{Phase 2 (12-month follow-up): text message survey}

Text messages were sent to service users at 12-month follow-up (see figure 2 for questions). A total of 335 messages were successfully delivered (phone numbers were available and in service). Of those successfully delivered, 77 participants returned a full survey. Over half of the retained participants reported that they had quit smoking tobacco $(n=42,55 \%$ of those retained). Figure 3 shows the outcomes all 77 participants. Those who had quit tobacco are shown in three categories: $17 \%$ of the sample (of 77 ) were not smoking and not using an e-cigarette (i.e. given up nicotine entirely); $4 \%$ were using e-cigarettes without nicotine; $34 \%$ were still using an e-cigarette with nicotine. Those who were still smoking tobacco were either using both tobacco and e-cigarettes (18\%) or using tobacco only (27\%).

Table 4 shows the 77 participants who were still engaged at 12-month follow-up, their status from phase one, and their status at phase two. There was significant variation between categories: the most likely group to have quit at 12 months were those who were confirmed (negative for carbon monoxide) to have quit at four weeks (23 quit, 3 not quit); those who were unconfirmed but self-reported as quit at 4 weeks were less likely to have quit, followed by those who were lost to follow-up at 4 weeks, and finally those who were still smoking at 4 weeks $\left(\chi^{2}=23.18, p<.001\right)$. Service users who used e-cigarettes as well as normal cigarettes reported smoking statistically significantly fewer cigarettes $(8.7)$ than those who just smoked cigarettes $(14.4)(F=5.79, d f=1,33, p=0.022)$.

Table 4

Twelve month outcome compared to outcomes at four weeks

\begin{tabular}{|llcccc|}
\hline Four week outcome & \multicolumn{4}{l}{ Outcome at 12 months } & Total \\
\cline { 2 - 6 } & \multicolumn{2}{c}{ Not quit } & \multicolumn{3}{c|}{ Self-reported quit } \\
\cline { 2 - 6 } & $\mathbf{n}$ & $\%$ & $\mathbf{n}$ & $\%$ & \\
\hline Lost to follow-up & 10 & 28.6 & 6 & 14.3 & $\mathbf{1 6}$ \\
\hline Not quit & 17 & 48.6 & 5 & 11.9 & $\mathbf{2 2}$ \\
\hline Self-reported quit & 5 & 14.3 & 8 & 19.0 & $\mathbf{1 3}$ \\
\hline CO-Confirmed quit & 3 & 8.6 & 23 & 54.8 & $\mathbf{2 6}$ \\
\hline Total & $\mathbf{3 5}$ & $\mathbf{1 0 0}$ & $\mathbf{4 2}$ & $\mathbf{1 0 0}$ & $\mathbf{7 7}$ \\
\hline
\end{tabular}

Among service users who were still smoking cigarettes, but not using e-cigarettes, the most common reason for people giving up their e-cigarettes was due to problems with the equipment, including procuring and using it $(n=7)$. This was followed by not liking the taste (5), the e-cigarette breaking (4) and it causing physical symptoms, e.g. chapped lips or coughing (3). Other reasons for giving up e-cigarettes included bad press (1), poor quality of the product (1), and the expense (1). Among service users who were no longer using either cigarettes or e-cigarettes, the most common reason for no longer using their e-cigarettes was that they had used them to quit cigarettes and now no longer needed them ( $n=5)$. Other reasons included not liking them (2), losing them ( 1 ), and claiming never to have had one (2).

\section{Phase 2 interviews with service users and providers at 12 months}

As part of the 12-month evaluation, 15 interviews were held with service users and four with pharmacists. The participants spoke positively about the intervention, especially the support from pharmacists and the quality of the e-cigarettes. They also spoke about the connection between stress and smoking, and this related to the ongoing Covid-19 pandemic and associated restrictions. Pharmacists spoke about their satisfaction with the intervention and that they felt it raised the profile of smoking cessation. They felt that the use of pharmacies was a useful way to access people in the local community, and they also described some problems related to the devices and their supply to the pharmacies. For the purpose of this analysis, the focus will be on new or different themes from those that were identified in phase one.

\section{Stress, smoking, and Covid-19}

As described in the phase one analysis, participants reported that smoking was related to stress. During times of stress there is a greater need to smoke, or cravings are more difficult to ignore. For some, this meant switching back to tobacco from e-cigarettes, at least for a short time. Several participants reported 
successfully swapping to e-cigarettes for a considerable amount of time as a result of taking part in the intervention, but then relapsing and returning to smoking tobacco due to stress.

"The fact that I was stressed out that's why I went back to the cigs, because I did it really well for about 3 months, and then, I was under a lot of stress and then I just went back to cigs." (Female, Smoker)

During the period between the phase one and phase two analyses (approximately June 2019 to June 2020), there were considerable social changes in the UK, which were related to the Covid-19 pandemic. Colloquially referred to as 'lockdown', millions of people were asked to stay in their homes and were only allowed out for essential reasons. These altered living arrangements led to a documented increase in stress and reduced health and wellbeing generally ${ }^{31}$. The participants in this study spoke in depth about the Covid-19 pandemic having a negative effect on their smoking behaviour. Feedback from some participants described how lockdown posed additional challenges in their efforts to quitting smoking due to increased stress, worry and isolation. This resulted in some participants smoking more than usual while others stopped using their e-cigarettes altogether and returned to smoking cigarettes.

"Well I am back smoking now, and I am smoking quite a lot and I am not liking it, I am really scared now I am smoking. I was ok for a bit and then lockdown happened and then just probably changes in life I would say, with what was happening. Stressing." (Female, Smoker)

\section{Pharmacies as an effective community hub for smoking cessation interventions}

Pharmacists reiterated the positive benefits of using their services as part of community health interventions, and especially for those involving smoking cessation. The combination of pharmacy advice and support along with free provision of the devices was seen as an effective strategy for smoking cessation. This specific intervention was seen as having raided the profile of smoking cessation in the local area, and especially in relation to e-cigarettes. Apparently there had been some negative impressions of e-cigarettes - some people saw them as dangerous and scary - which pharmacists were able to counter with evidence-based advice.

"I think the other good thing from this... is that I think it...maybe raised the profile of e-cigs...because I think when they see it sort of coming from a pharmacy and from you know like the smoking clinic teams as well, it is kind of saying you know e-cigs are safe to use those. I think when they first came out they were sort of seen as maybe a bit dangerous weren't they, and...some of the models that were out weren't necessarily, they got a bit of bad press, so I think that has done good for e-cig in general yes. For raising its profile so, yes." (Pharmacy 1)

Pharmacists' positions as primary healthcare providers within local communities puts them in a unique position to be able to give advice daily and locally to high numbers of local people. There was a strong sense of connection to the local community and pharmacists were keen to give as much help and support as possible to service users during their efforts to quit smoking. They reported that they wanted to create a sense of 'togetherness' between service provider and service user, to help build trusting relationships with participants and remove any barriers that may have prevented them from seeking help during the intervention.

"Yeah I think they think pharmacy is a good idea to come to. To come and talk or just get some more advice from the pharmacist as well, whatever it is." (Pharmacy 4)

\section{Discussion}

The aim of this intervention was to encourage smokers to quit tobacco by switching to e-cigarettes with the support and advice of their local pharmacist. The aims of this evaluation were to assess the efficacy of the intervention in terms of number of quitters and reduced smoking among smokers, and to explore the experiences of service users and providers (pharmacists). Quantitative and qualitative assessments were commenced at three months (phase 1 ) and 12 month follow-up (phase 2). Both phases showed evidence that service users reduced their use of tobacco. A total of 871 people took part in the scheme, with $18 \%$ (confirmed) to $26 \%$ (confirmed and unconfirmed) of the sample no longer using tobacco at 3 months, and $55 \%$ of those retained in the study having quit tobacco at 12 -month follow-up ( $5 \%$ of full sample). Service users and providers spoke positively about their experiences, while also providing useful descriptions of factors associated with the struggle of nicotine dependency.

Taken in context with the previously published Greater Manchester pharmacy-led e-cigarette intervention ${ }^{29}$, these studies show that e-cigarettes appear to be an agreeable and effective alternative to tobacco, which can aid smoking cessation, especially when combined with pharmacy support. The qualitative findings in this report suggest that the pharmacy support was an important factor, and this may be largely due to the fact that pharmacies represent a bridge between primary care and the local community. Since the previous Greater Manchester intervention concluded that longer-term follow-up was required, this study included assessments at both 3-months and 12-months. However, the majority of participants were not engaged with the study at 12-month follow-up, which diminished the power of the long-term quantitative assessment. Only 77 out of 871 (8.8\%) participants provided any data at 12 months. Further studies should be designed to overcome this limitation, perhaps by conducting a 6-month follow-up or by offering an incentive.

\section{Conclusion}

Based on the phase one data, up to one quarter of people given this e-cigarette intervention managed to stop smoking tobacco in four weeks. A further $61 \%$ of those who were still smoking had reduced their intake by five cigarettes per day. It is difficult to draw firm conclusions from the phase two data due to low levels of participant engagement at that stage, but the experiences of both service users and providers over both phases was positive. Although more data are required on long-term health effects, e-cigarettes appear to be an agreeable and effective smoking cessation therapy and interventions similar to this one are recommended. 


\section{Declarations}

\section{Ethical approval and consent to participate}

All participants provided their informed consent to participate. All methods were carried out in accordance with relevant guidelines and regulations. All experimental protocols were approved by the University of Salford Research Ethics Committee (HSR1819-064 - 22nd March 2019).

\section{Consent for Publication}

Consent to publish was not required since no personal details are reported.

\section{Conflict of Interest}

The authors state that they have no competing interests to declare.

\section{Acknowledgement}

The authors are grateful to the study participants including service providers and service users for taking part. We thank Trafford Metropolitan Borough Council for funding the study.

\section{Funding}

Funding for the project was provided by Trafford Metropolitan Borough Council.

\section{Author Contribution}

The study was designed and conducted by MC, LH and PAC. Interviews were carried out by MC and LH. Qualitative analysis was carried out by MC, LH and ADP. Quantitative analysis was carried out by PAC and ADP. The manuscript was prepared by ADP with input from MC, LH and PAC. All authors approved the manuscript prior to submission.

\section{Availability of data and materials}

The anonymised datasets used and/or analysed during the current study are available from the corresponding author on reasonable request.

\section{References}

1. World Health Organisation. Tobacco, https://www.who.int/news-room/fact-sheets/detail/tobacco (2020, May 27).

2. Public Health England. Smoking and tobacco: applying All Our Health, https://www.gov.uk/government/publications/smoking-and-tobacco-applying-allour-health/smoking-and-tobacco-applying-all-our-health (2020, June 16).

3. Siahpush M, Borland R and Scollo M. Smoking and financial stress. Tobacco control 2003; 12: 60-66.

4. Polosa R, Rodu B, Caponnetto P, et al. A fresh look at tobacco harm reduction: the case for the electronic cigarette. Harm reduction journal 2013 ; 10 : $1-11$.

5. World Health Organisation. Tobacco: Health benefits of smoking cessation, https://www.who.int/news-room/q-a-detail/tobacco-health-benefits-ofsmoking-cessation (2020, February 25).

6. Fong GT, Hammond D, Laux FL, et al. The near-universal experience of regret among smokers in four countries: findings from the International Tobacco Control Policy Evaluation Survey. Nicotine \& Tobacco Research 2004; 6: S341-S351.

7. Twyman L, Bonevski B, Paul C, et al. Perceived barriers to smoking cessation in selected vulnerable groups: a systematic review of the qualitative and quantitative literature. BMJ open 2014; 4.

8. O'Leary R and Polosa R. Tobacco harm reduction in the 21st century. Drugs and Alcohol Today 2020.

9. Wiltshire S, Bancroft A, Parry O, et al. 'I came back here and started smoking again': perceptions and experiences of quitting among disadvantaged smokers. Health Education Research 2003; 18: 292-303.

10. Office for National Statistics. Adult smoking habits in the UK: 2019,

https://www.ons.gov.uk/peoplepopulationandcommunity/healthandsocialcare/healthandlifeexpectancies/bulletins/adultsmokinghabitsingreatbritain/20 (2020, July 7).

11. Kock L, Brown J, Hiscock R, et al. Individual-Level behavioural smoking cessation interventions tailored for disadvantaged socioeconomic position: a systematic review and meta-regression. The Lancet Public Health 2019; 4: e628-e644.

12. Aveyard P and Raw M. Improving smoking cessation approaches at the individual level. Tobacco control 2012; 21: 252-257.

13. Laniado-Laborín R. Smoking cessation intervention: an evidence-based approach. Postgraduate medicine 2010; 122 : 74-82.

14. Stead LF, Buitrago D, Preciado N, et al. Physician advice for smoking cessation. Cochrane database of systematic reviews 2013. 
15. Lancaster T and Stead LF. Individual behavioural counselling for smoking cessation. Cochrane database of systematic reviews 2017.

16. Kaur K, Kaushal S and Chopra SC. Varenicline for smoking cessation: A review of the literature. Current therapeutic research 2009; 70: 35-54.

17. Lemmens V, Oenema A, Knut IK, et al. Effectiveness of smoking cessation interventions among adults: a systematic review of reviews. European journal of cancer prevention 2008; 17: 535-544.

18. Saba M, Diep J, Saini B, et al. Meta-analysis of the effectiveness of smoking cessation interventions in community pharmacy. Journal of clinical pharmacy and therapeutics 2014; 39: 240-247.

19. Kalkhoran S and Glantz SA. E-cigarettes and smoking cessation in real-world and clinical settings: a systematic review and meta-analysis. The Lancet Respiratory Medicine 2016; 4: 116-128.

20. Notley C, Ward E, Dawkins L, et al. The unique contribution of e-cigarettes for tobacco harm reduction in supporting smoking relapse prevention. Harm reduction journal 2018; 15: 1-12.

21. Hajek P, Phillips-Waller A, Przulj D, et al. A randomized trial of e-cigarettes versus nicotine-replacement therapy. New England Journal of Medicine 2019; 380: 629-637.

22. Glantz SA and Bareham DW. E-cigarettes: use, effects on smoking, risks, and policy implications. Annual review of public health 2018; 39 : 215-235.

23. Worku D and Worku E. A narrative review evaluating the safety and efficacy of e-cigarettes as a newly marketed smoking cessation tool. SAGE open medicine 2019; 7: 2050312119871405.

24. Yan XS and D'Ruiz C. Effects of using electronic cigarettes on nicotine delivery and cardiovascular function in comparison with regular cigarettes. Regulatory Toxicology and Pharmacology 2015; 71: 24-34.

25. Hershberger AR, Karyadi KA, VanderVeen JD, et al. Beliefs about the direct comparison of e-cigarettes and cigarettes. Substance use \& misuse 2017; 52: 982-991.

26. Foulds J, Veldheer S and Berg A. Electronic cigarettes (e-cigs): views of aficionados and clinical/public health perspectives. International journal of clinical practice 2011; 65: 1037-1042.

27. Strick K. E-Cigarettes: time to realign our approach. Lancet 2019; 394: 10.1016.

28. GMHSCP. Making Smoking History: A Tobacco Free Greater Manchester, https://www.gmhsc.org.uk/wp-content/uploads/2018/04/Tobacco-Free-GreaterManchester-Strategy.pdf (2017).

29. Coffey M, Cooper-Ryan A, Houston L, et al. Using e-cigarettes for smoking cessation: evaluation of a pilot project in the North West of England. Perspectives in public health 2020; 140: 351-361.

30. Braun V and Clarke V. Using thematic analysis in psychology. Qualitative research in psychology 2006; 3: 77-101.

31. Evandrou M, Falkingham J, Qin M, et al. Changing living arrangements and stress during Covid-19 lockdown: Evidence from four birth cohorts in the UK. SSM-population health 2021; 13: 100761.

\section{Figures}

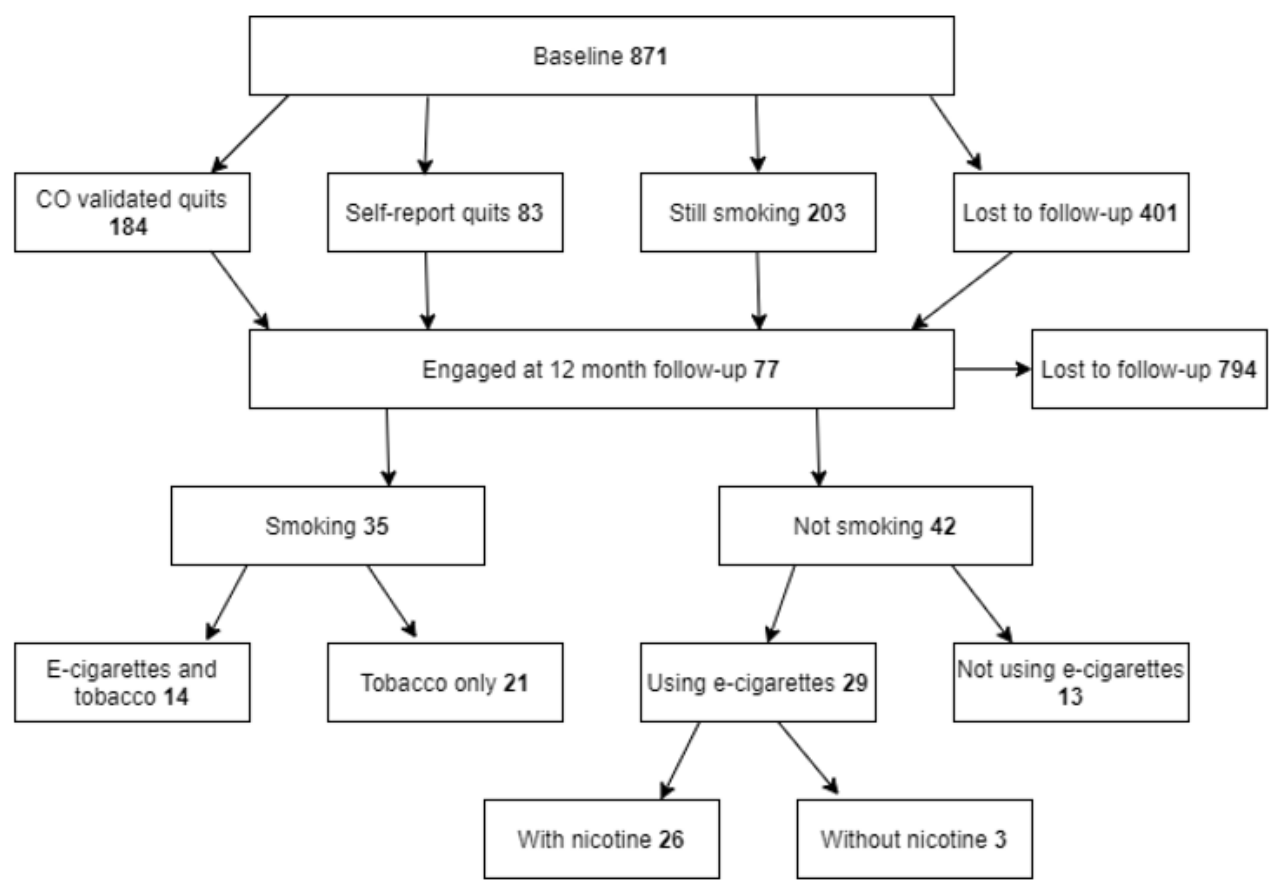

\section{Figure 1}

Flow chart of participants through the first and second stages 
Opening Message

Hi, this is Trafford Public Health team. You took part in the Trafford E-Cigarette pilot last year. We'd really welcome your feedback via a short survey which contains a maximum of 9 brief questions we'd like you to answer; you will shortly receive the 1st question from 0000000000 .

Questions

1. This survey consists of 9 short SMS questions and will take a couple of minutes to complete. Please reply Yes to take part and the survey will begin.

1. If no-close survey

2. If yes - move onto question 2

2. Are you still smoking normal cigarettes? Reply Yes or No.

1. If no - move to question 4

2. If yes - move to question 3

3. Can you tell us how many normal cigarettes you are smoking each day? Reply using only a number.

1. Free text for providing a number. Move to question 4

4. Are you still using an e-cigarette? Reply Yes or No.

1. If no-move onto question 6

2. If yes - move onto question 5

5. What strength of nicotine (e.g. $0 \%(0 \mathrm{mg} / \mathrm{ml}), 0.8 \%(0.8 \mathrm{mg} / \mathrm{ml})$ do you usually use in your e-cigarette liquid now? Reply using only a number.

1. Free text for providing a number. Move to question 7

6. Can you tell us why you stopped using your e-cigarette?

1. Free text to provide reason - move onto question 7.

7. Have you received any further support (after the initial first 4 weeks) with regards to quitting smoking? Reply Yes or No.

1. If no - move to question 9

2. If yes - move to question 8

8. Where or who did you get this support from with regards quitting smoking?

1. Free text to provide source of support - move to question

9. Would you be happy for us to call you and take part in a short telephone interview to discuss this further? Please reply Yes or No.

\section{Figure 2}

Text message survey options (phase 2)

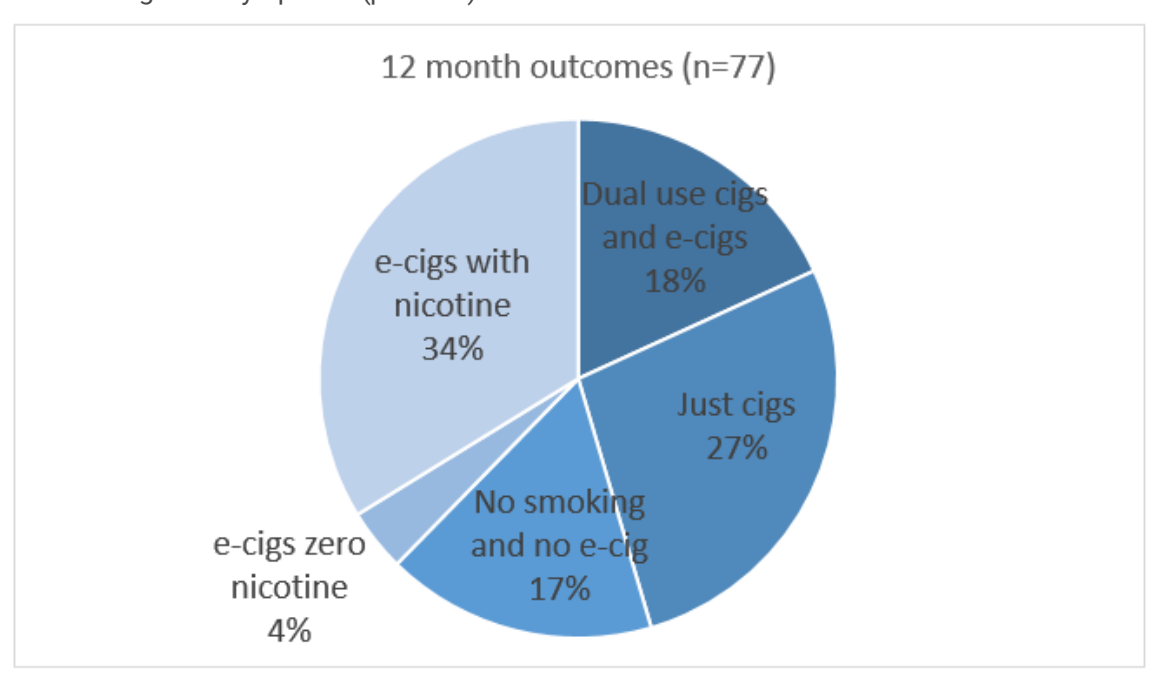

\section{Figure 3}

Outcome of text message survey at 12 months (phase 2) 